\title{
Single-Handed Cursor Control Technique Optimized for Rear Touch Operation and Its Usability
}

\author{
Yoshikazu Onuki \\ Department of Information Processing \\ Tokyo Institute of Technology \\ Yokohama, Japan 226-8503
}

\author{
Itsuo Kumazawa \\ Imaging Science and Engineering Laboratory \\ Tokyo Institute of Technology \\ Yokohama, Japan 226-8503
}

\begin{abstract}
To improve single-handed operation of mobile devices, the use of rear touch panel has potential for user interactions. In this paper, a basic study of operational control simply achieved through drag and tap of the index finger on a rear touch panel is conducted. Since a user has to hold the handheld device firmly with the thumb and fingers, a movable range of the tip of an index finger is limited. This restriction requires a user to perform several times of dragging actions to reach a cursor to the long distance target. Considering such kinematic restriction, a technique optimized for rear operation is proposed, wherein not only the position but also the velocity of fingertip movement is regarded. Movement time, the number of dragging operation, and the throughputs of the proposed technique have been evaluated in comparison with the generic technique using Fitts's law. Experiments have been conducted to perform the target selection in the form of reciprocal 1D pointing tasks with ten participants. The combinations of two ways of holding the device (landscape and portrait) and two directions of dragging (horizontal and vertical) are considered. As a result, the proposed technique achieved the improvements of from 5 to $13 \%$ shorter movement time, from 20 to $40 \%$ higher throughputs and no deterioration of the number of dragging even for the longer distance targets. In addition, the further analysis addressed that there exists the advantageous combinations of the way of holding and the direction of dragging, which would be beneficial for better design of single-handed user interactions using rear touch.
\end{abstract}

Keywords-Rear touch; cursor control; mobile device; singlehanded; Fitts's law

\section{INTRODUCTION}

Many types of mobile devices have appeared on the market in recent years. Such devices satisfy various inherent needs and diverse lifestyles. Mobile game consoles, which have a longer history than cell phones, are also enlivening the market. In general, two hands are required to use such devices; the device is held in one hand and operated with the other. However, sometimes only one hand is free to operate the device. In addition, as display sizes increase, single-handed thumb-touch operations become more difficult. To address these issues, we have considered the use of rear touch and proposed the technique to fully utilize the limited movable range of the tip of an index finger. Using a portable gaming device with a rear touch panel, we demonstrate a practical single-handed input technique for mobile devices. The purpose of this study is not to compare the proposed technique to existing bimanual methods, such as generic front touch operations. The benefit of the use of rear touch is that it works sufficiently even when the generic modes of operation are not stable or available Another benefit is no blockage of the display. In generic front touch operation, occlusion and selection point ambiguity are problematic, and the use of rear touch has potential to be the solution.

The remainder of this paper is organized as follows. Related work is presented in Section II. A design for rear touch operation, which addresses the generic technique and the proposed rear touch oriented technique, is described in Section III. Methods of experiments are presented in Section IV, and results of performance evaluation are shown in Section V. Moreover, discussions are presented in Section VI, and conclusions and future works are given in Section VII.

\section{RELATED WORK}

\section{A. Rear Touch}

In recent touchscreen-based mobile devices, there is a challenging trade-off between visual expressivity and ease of interaction. Information-rich interfaces on a small display result in a target size that requires thumb and finger operations. Occlusion and selection point ambiguity are problematic, and a number of studies have addressed these problems [1], [2], [3], [4]. On the other hand, rear touch technology is a recent development, and such technology would greatly benefit tabletbased devices where the front touch panel occupies most of the surface area and leaves little space for other input methods. Practical research for this technology is limited [5]. [6], [7]. We find enhanced studies for the simultaneous use of the front and the back of the device [8], [9], [10], and the text input [11], [12]. Reference [13] conducted the performance analysis of the rear touch and found that the index finger performed well on the small-sized display device. Recently, [14], [15] explored the relationship between hand grip from the back of the device and user interactions. With regard to single-handed rear touch panel operation, different ways to hold hand-held devices have been investigated, and five spontaneous ways to hold tablets have been described [16] and analyzed using a kinematic chain model [17]. A kinematic chain approach is appropriate for considering holding methods that involve frame, support, and interact in the kinematic chain. However, in this study, we do not discuss different ways to hold mobile devices in detail. Rather, we evaluate two cursor control techniques in relation to particular holding methods, landscape and portrait orientations. At present, most hand-held tablets are thin rectangular-shaped 


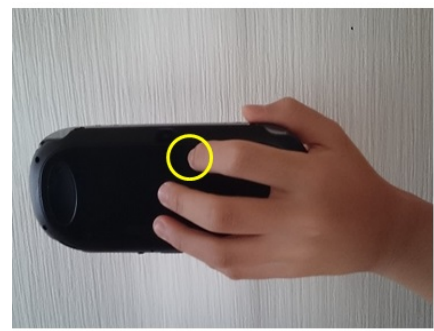

(a) Landscape

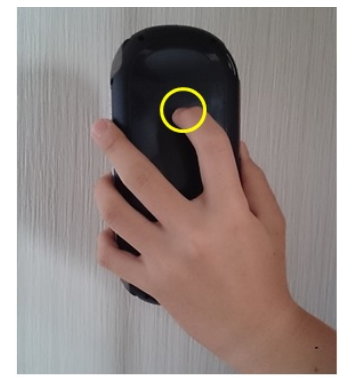

(b) Portrait
Fig. 1: Ways of holding the device

devices weighing $250-600 \mathrm{~g}$ with a 7-10 inch display. Smart phones, which are also rectangular, typically weigh 100-200 g with a $4-5.5$ inch display. The trend is toward larger displays and reduced weight. In general, devices that weigh less are easier to hold for a long time with one hand and can be held more easily in various ways. We assume that hand-held devices that weigh up to $200 \mathrm{~g}$ will not result in user stress. However, this assumption should be carefully examined from various perspectives, and will be considered in future studies. In this study, we define two particular holding methods and evaluate two cursor control techniques using a hand-held gaming device that weighs $219 \mathrm{~g}$.

\section{B. Target Selection}

In this study, the performance of two cursor control techniques are evaluated using Fitts's classic 1D reciprocalpointing paradigm [18] as modified by MacKenzie [19]. Although it dealt with traditional pointing devices, such as a mouse and a stylus, we applied this evaluation norm for these rear touch techniques. Moreover, it does not fully indicate the usability or the practicability of systems; nevertheless, we applied Fitts's law to assess the fundamental performance. Fitts's law states that target acquisition time, or movement time $(M T)$, in graphical user interfaces is almost entirely determined by the ratio of target distance $D$ and target width $W$ as follows:

$$
M T=a+b \log _{2}(D / W+1),
$$

where $a$ and $b$ are constants determined through linear regression. The expression $\log _{2}(D / W+1)$ defines what Fitts referred to as the index of difficulty (ID) and carries the unit "bits." If $M T$ is measured in seconds, then the unit for $a$ is "s" and the unit for $b$ is "s/bit." The reciprocal of $b$ defines what Fitts termed as the index of performance $(I P)$ in "bits/s." $I P$ is the rate of human information processing for the movement task under investigation and is referred to as bandwidth or throughput.

\section{DESIGN FOR REAR TOUCH OPERATION}

Two ways of holding tablets are considered. Fig. 1 shows the ways of holding the device in landscape and portrait orientation, respectively. The yellow circles in Fig. 1 indicate movable ranges of the tip of an index finger. In both orientations, they are limited in the size of approximately from 10 to $15 \mathrm{~mm}$ (around $0.5 \mathrm{inch}$ ) in diameter. On the other hand, the display size of hand-held devices is fairly large in size of from 4 to $10 \mathrm{inch}$. Therefore, an input within a limited range has to control a cursor which covers from 8 to 20 times wider range on the display.

\section{A. Generic Technique}

In this study, two cursor control techniques using rear touch are evaluated. One is the generic technique known as the standard touch pad control, wherein touch movement is linearly applied for cursor movement. The mapping algorithm from the touch position to the cursor position is expressed as follows:

$$
q(k)-q(0)=M_{p}(p(k)-p(0)),
$$

where $k>0$ is the sampling number (sampling frequency was $60 \mathrm{~Hz}$ in the implementation), $p(k) \in R^{2 \times 1}$ is the current touch position, $p(0)$ is the start position of dragging, $q(k) \in R^{2 \times 1}$ is the current cursor position, $q(0)$ is the initial cursor position associated with $p(0)$, and $M_{p} \in R^{2 \times 2}$ is the appropriate constant diagonal matrix. Note that $p(k)$ exists in a rear touch pad coordinate system, and $q(k)$ exists in a display coordinate system. The action of repeating dragging achieves the long distance movement of a cursor. In the implementation design, we used

$$
M_{p}=\left(\begin{array}{cc}
6 & 0 \\
0 & 6
\end{array}\right)
$$

in consideration with a balance of the gain of cursor movement and the cursor positioning accuracy.

\section{B. Rear Touch Oriented Technique}

Considering the kinematic restriction of finger movement on the rear face, a technique optimized for rear operation is proposed. The proposed technique achieves long distance cursor movement by small distance fingertip movement. Here, a velocity of fingertip movement is considered so that the faster dragging leads to the longer distance movement of a cursor. The proposed mapping algorithm from the touch position to the cursor position is expressed as follows:

$$
q(k)-q(0)=M_{p}(p(k)-p(0))+M_{v}(p(k)-p(k-1))^{2},
$$

where $M_{v} \in R^{2 \times 2}$ is the appropriate constant diagonal matrix. In the implementation design, we used

$$
M_{v}=\left(\begin{array}{cc}
1 / 288 & 0 \\
0 & 1 / 288
\end{array}\right) \text {. }
$$

In addition, we applied the limiter for $p(k)-p(k-1)$ to avoid the excessively fast movement of a cursor, which was set as approximately $0.53 \mathrm{~mm} / \mathrm{sample}$ in the implementation.

\section{METHOD}

\section{A. Apparatus}

Implementation and evaluation were conducted on a PS Vita. The PS Vita has a front 5 inch organic light-emitting diode touch panel display with a resolution of $960 \times 544$ pixels, which corresponds to a single pixel size of approximately $0.115 \mathrm{~mm}$. On the back, the PS Vita has a capacitivesensing touch panel with a maximum of six touch points. The experimental application was run at the sampling rate of 60 frames/s. 


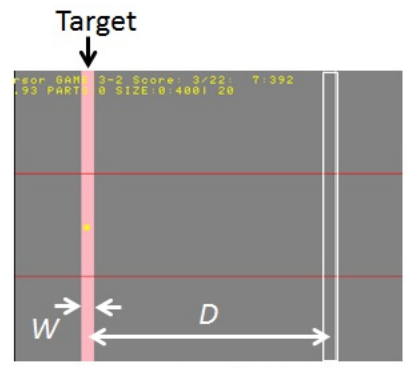

(a) Target in left side

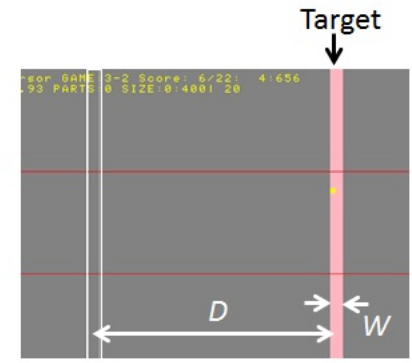

(b) Target in right side
Fig. 2: Target selection task $(D=400, W=20)$

\section{B. Participants}

Ten volunteers ( 4 female, 6 male; $14-50$ years) participated. All were right-handed, and held the device and operated rear touch with their left hand. All participants regularly use smartphones and/or tablet devices.

\section{Procedure}

The performance of the target selection operation was evaluated using Fitts's law 1D reciprocal-pointing paradigm. The 1D pointing task required participants to select two fixedsized targets repeatedly in succession (Fig. 2). The targets were rendered as solid rectangles equidistant from the center of the display in opposite directions along the horizontal or vertical axis. The targets to be selected were colored red, and they appeared one after another on opposite sides of the display. In addition, the target color changed to light pink when the cursor was on the target. When participants correctly selected a target, the current target briefly became white and disappeared; then, the next target would appear on the opposite side of the display. Participants moved a cursor by performing drag operation on a rear touch panel (repeatedly, if necessary), and tapped to accomplish a selection. A task was continued until participants completed a selection successfully, and therefore, a faulty selection did not occur (no errors). Instead of error rates, we counted and evaluated the number of dragging actions per task.

\section{Independent Variables}

The performance of the generic technique and the proposed technique was evaluated. We specifically focused on the way of holding the handheld device and the direction to move the tip of an index finger. Therefore, the independent variables were the cursor control technique $T E C$, the way of holding the device $H L D$, the direction of dragging $D I R$, the target distance $D$, and the target width $W$.

\section{E. Dependent Variables}

The main dependent variable is $M T$, which is defined as the time taken to move to and select the active red target. The other main dependent variable is the number of dragging operation $N U M$.
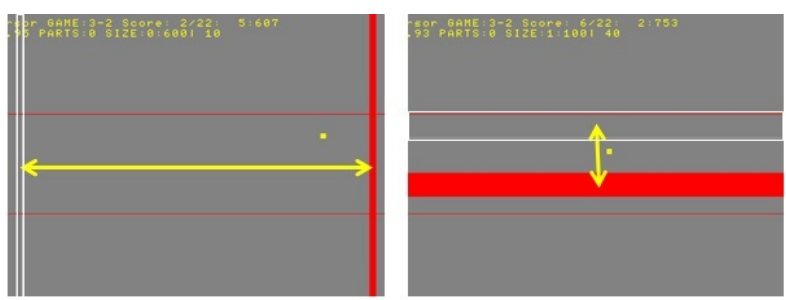

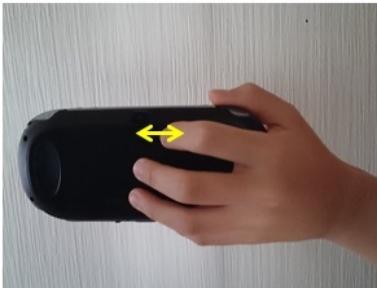

(a) Landscape $x$ Horizontal $(D=600, W=20)$
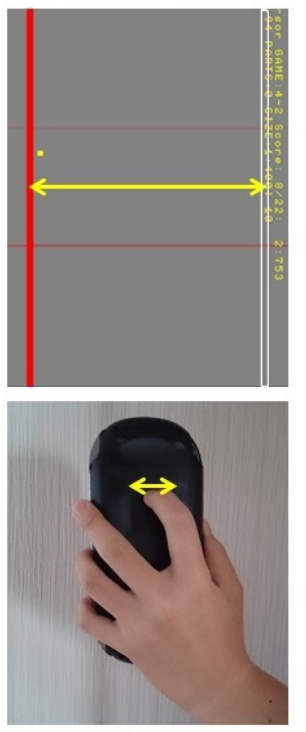

(c) Portrait $x$ Horizontal $(D=400, W=10)$

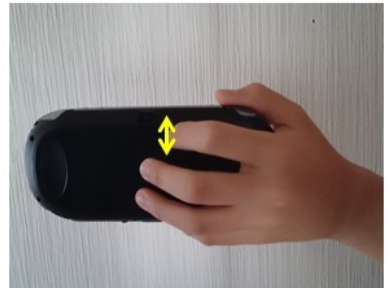

(b) Landscape $x$ Vertical $(D=100, W=40)$
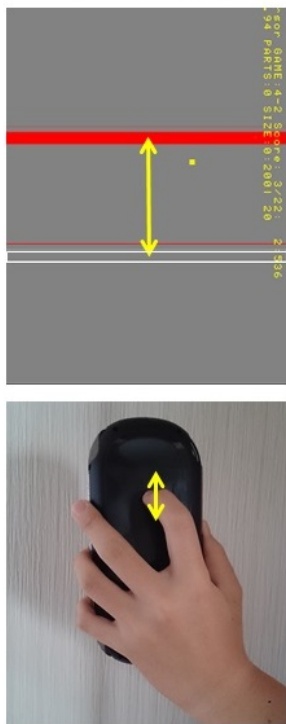
$(D=200, W=20)$ (d) Portrait $x$ Vertical

Fig. 3: The directions of fingertip movement and associated cursor movement for four models $(H L D \times D I R)$

\section{F. Design}

The independent variables were the cursor control technique TEC ("G"eneric technique, "A"ccelerated technique), the way of holding HLD ("L"andscape, "P"ortrait), the direction of dragging $D I R$ ("H"orizontal, "V"ertical), the target distance $D(100,200,400,600$ pixels $)$, and the target width $W$ (10,20,40 pixels). The target distance $D=600$ was available only for the models of $\mathrm{LH}(H L D=\mathrm{L}, D I R=\mathrm{H})$ and PV $(H L D=\mathrm{P}, D I R=\mathrm{V})$ due to the display size limitation. Here, we express each factor for TEC, HLD and DIR as the first character of each word, hence, $H L D=\mathrm{L}$ means $H L D=$ Landscape. A fully crossed design resulted in 84 combinations of $T E C, H L D, D I R, D$, and $W$. Fig. 3 shows images of four models $\mathrm{LH}, \mathrm{LV}, \mathrm{PH}$ and $\mathrm{PV}(H L D \times D I R)$. The lower photographs show the way of holding the device in each orientation, wherein the directions of movement of the tip 


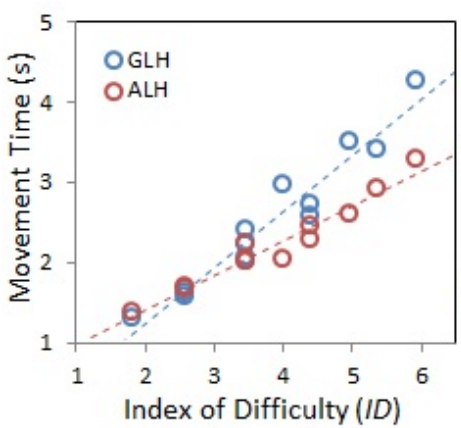

(a) GLH v.s. ALH

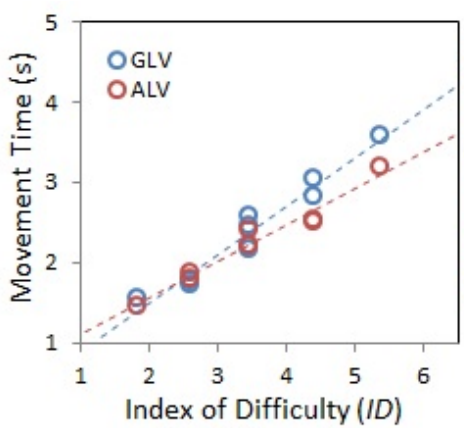

(b) GLV v.s. ALV

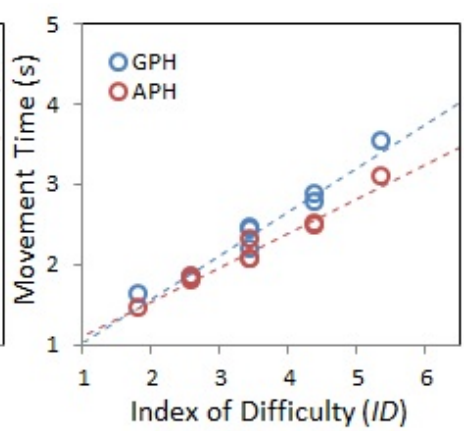

(c) GPH v.s. APH

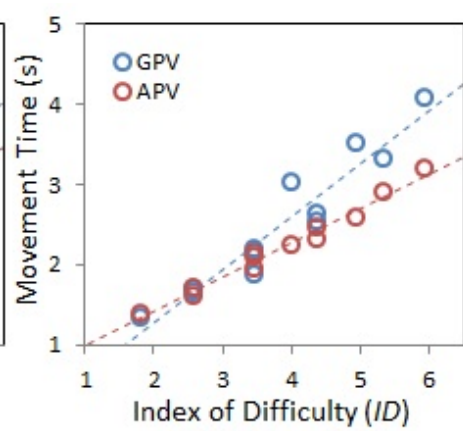

(d) GPV v.s. APV

Fig. 4: Movement time by $I D$ for eight models

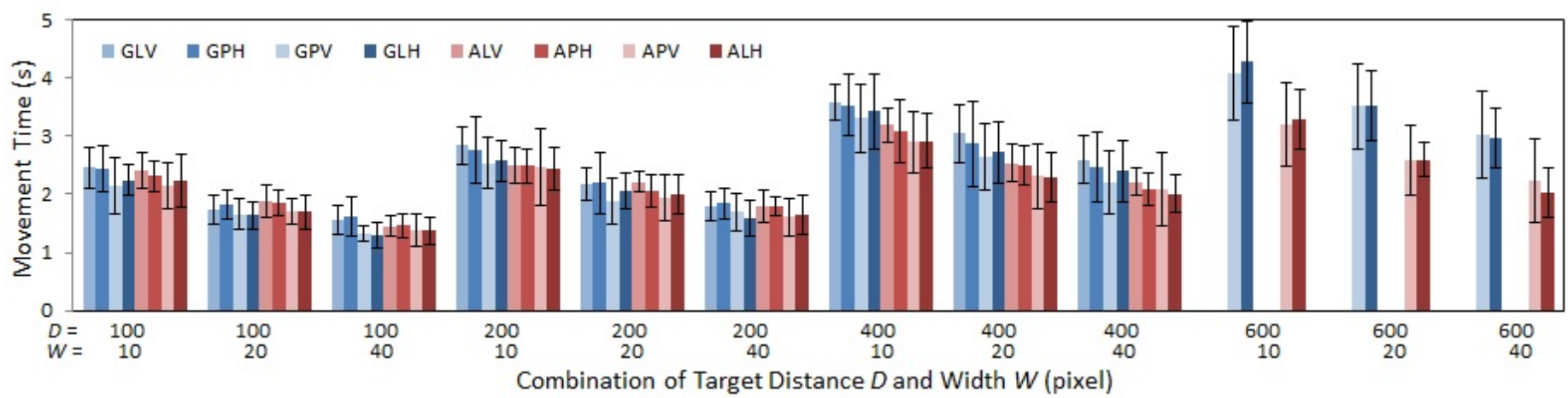

Fig. 5: Movement time by $W$ and $D$ for eight models

of an index finger are indicated as yellow arrows. The upper images show the display image of the experimental application, wherein the targets are colored red and the directions of cursor movement are indicated as yellow arrows.

The experiment was organized into four sessions for eight models $M D L$ (GLH, GLV, ALH, ALV, GPH, GPV, APH, APV). The participants were separated into two teams (five participants for each). For each team, sessions were organized as follows:

$\begin{array}{lcc} & \text { Team 1 } & \text { Team 2 } \\ \text { Session 1: } & \text { GLH } \rightarrow \text { ALH } & \text { GPV } \rightarrow \text { APV } \\ \text { Session 2: } & \text { ALV } \rightarrow \text { GLV } & \text { APH } \rightarrow \text { GPH } \\ \text { Session 3: } & \text { GPV } \rightarrow \text { APV } & \text { GLH } \rightarrow \text { ALH } \\ \text { Session 4: } & \text { APH } \rightarrow \text { GPH } & \text { ALV } \rightarrow \text { GLV }\end{array}$

Here, GLH $\rightarrow$ ALH means that participants attempt all trials of GLH first and those of ALH second in a session. Each participant performed the trials in a single session that lasted approximately $60 \mathrm{~min}$. Prior to starting the session, the participants were allowed $5 \mathrm{~min}$ of practice. The session was broken up by $M D L$, with nine or twelve trial sets $(D \times W)$ completed for each $M D L$. The nine or twelve trial sets were separated into three blocks to counterbalance order, fatigue, and the effects of practice.

$$
\begin{aligned}
\text { Block 1: } & D=100, W=10 / D=200, W=20 / \\
& D=400, W=40 / D=400, W=10 \\
\text { Block 2: } & D=100, W=20 / D=200, W=40 / \\
& D=400, W=10 / D=400, W=20 \\
\text { Block 3: } & D=100, W=40 / D=200, W=10 / \\
D & =400, W=20 / D=400, W=40
\end{aligned}
$$

TABLE I: Regression Coefficients for Eight Models

\begin{tabular}{|l|c|c|c|c|}
\hline \multirow{2}{*}{ Model } & \multirow{2}{*}{$r^{2}$} & \multicolumn{3}{|c|}{ Regression Coefficients } \\
\cline { 3 - 5 } & & $\begin{array}{c}\text { Intercept } \\
\mathrm{a}(\mathrm{s})\end{array}$ & $\begin{array}{c}\text { Slope } \\
\mathrm{b} \text { (s/bit) }\end{array}$ & $\begin{array}{c}I P \\
\text { (bits/s) }\end{array}$ \\
\hline GLH & 0.9397 & 0.156 & 0.703 & 1.4 \\
\hline GLV & 0.9550 & 0.312 & 0.602 & 1.7 \\
\hline GPH & 0.9648 & 0.484 & 0.546 & 1.8 \\
\hline GPV & 0.9147 & 0.060 & 0.663 & 1.5 \\
\hline ALH & 0.9453 & 0.550 & 0.433 & 2.3 \\
\hline ALV & 0.9603 & 0.654 & 0.454 & 2.2 \\
\hline APH & 0.9636 & 0.684 & 0.429 & 2.3 \\
\hline APV & 0.9777 & 0.557 & 0.429 & 2.3 \\
\hline
\end{tabular}

Here, the trial sets of $D=600$ were conducted only for the four models GLH, GPV, ALH and APV. In each block, the participants completed trial sets for three or four combinations of $D$ and $W$ that were presented randomly. A 5 min break was taken between each block; therefore, there were six blocks of trials and five breaks. A trial set considered 12 selection tasks, i.e., 11 reciprocal movements between 12 targets. The data of the first two selection tasks were discarded.

\section{RESUlts}

$M T$ and $N U M$ for the eight models were evaluated. As $D=600$ was available only for the four models, we define two categories where the combinations of $M D L, D$ and $W$ 


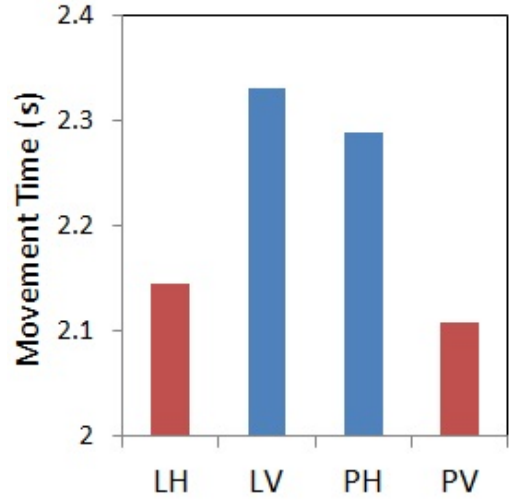

Fig. 6: $M T$ by $H L D \times D I R$
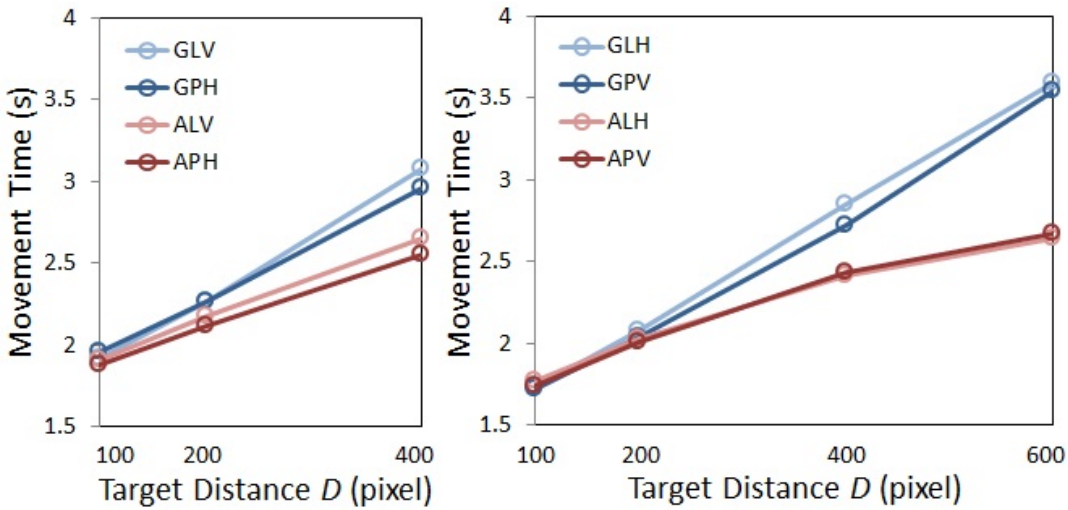

Fig. 7: $M T$ by $D$ for eight models are defined as follows:

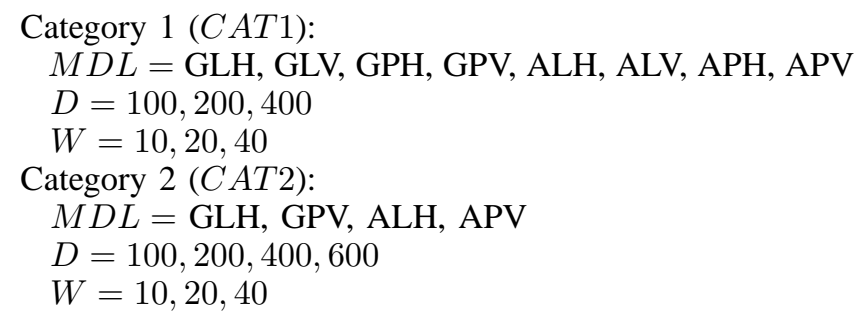

\section{A. Movement Time}

Fig. 4 plots $M T$ of the eight models as a function of $I D$. The results of linear regression analysis for each plot are given in Table I. The fits between the Fitts's law predictions and the data collected in the rear-touch-based cursor control operations yielded $r^{2}$ values above 0.91 . Moreover, from 20 to $40 \%$ improvements are found in throughputs $(I P)$ by using the proposed technique. The mean $M T$ in $C A T 1$ were $2.220 \mathrm{~s}$ for GLH, $2.419 \mathrm{~s}$ for GLV, 2.394 s for GPH, $2.151 \mathrm{~s}$ for GPV, $2.081 \mathrm{~s}$ for ALH, 2.244 $\mathrm{s}$ for $\mathrm{ALV}, 2.184 \mathrm{~s}$ for APH, and $2.061 \mathrm{~s}$ for APV. The mean $M T$ in $C A T 2$ were $2.563 \mathrm{~s}$ for GLH, $2.503 \mathrm{~s}$ for GPV, 2.221 $\mathrm{s}$ for ALH, and $2.214 \mathrm{~s}$ for APV. Fig. 5 shows all $M T$ plots by $D$ and $W$ for the eight models, wherein the models are roughly arranged in descending order for each TEC. Repeated measure analysis of variance in $C A T 1$ showed a significant main effect for TEC $\left(\mathrm{F}_{1,9}=49.62, \mathrm{p}=0.0001\right)$, $D\left(\mathrm{~F}_{2,18}=958.90, \mathrm{p}<0.0001\right)$, and $W\left(\mathrm{~F}_{2,18}=436.29\right.$, $\mathrm{p}<0.0001)$. The following interaction effects were observed: $H L D \times D I R\left(\mathrm{~F}_{1,9}=39.48, \mathrm{p}=0.0001\right)$. Fig. 6 shows $M T$ for LH, LV, PH and PV $(H L D \times D I R)$. Apparently, $M T$ of $\mathrm{LH}$ and PV is shorter than that of $\mathrm{LV}$ and $\mathrm{PH}$. This result implies that fingertip movement of the longitudinal direction would be easier than that of the transverse direction. Repeated measure analysis of variance in $C A T 2$ showed a significant main effect for TEC $\left(\mathrm{F}_{1,9}=280.72, \mathrm{p}<0.0001\right)$, $D\left(\mathrm{~F}_{3,27}=373.00, \mathrm{p}<0.0001\right)$, and $W\left(\mathrm{~F}_{2,18}=284.19\right.$, $\mathrm{p}<0.0001)$. The following interaction effects were observed: $T E C \times D\left(\mathrm{~F}_{3,27}=63.52, \mathrm{p}<0.0001\right)$ and $D \times W$ $\left(\mathrm{F}_{6,54}=8.53, \mathrm{p}<0.0001\right)$. Fig. 7 shows $M T$ by $D$ for the eight models. Evidently, $M T$ was improved by the proposed technique. As a result, the mean $M T$ by the proposed technique was from 5 to $9 \%$ shorter in $C A T 1$ and approximately
$13 \%$ shorter in $C A T 2$ than that of the generic technique. This tendency is particularly noticeable at $D=600$, so that more than $25 \%$ of improvements are observed.

\section{B. The Number of Dragging}

The mean $N U M$ in $C A T 1$ were 1.337 for GLH, 1.380 for GLV, 1.140 for GPH, 1.091 for GPV, 1.048 for ALH, 1.084 for ALV, 1.064 for APH, and 1.042 for APV. The mean $N U M$ in $C A T 2$ were 1.563 for GLH, 1.336 for GPV, 1.063 for ALH, and 1.054 for APV. Fig. 8 shows all $N U M$ plots by $D$ and $W$ for the eight models. Repeated measure analysis of variance in CAT1 showed a significant main effect for TEC $\left(\mathrm{F}_{1,9}=122.10, \mathrm{p}<0.0001\right)$, $H L D \quad\left(\mathrm{~F}_{1,9}=150.21, \quad \mathrm{p}<0.0001\right), \quad D \quad\left(\mathrm{~F}_{2,18}=198.48\right.$, $\mathrm{p}<0.0001)$, and $W\left(\mathrm{~F}_{2,18}=116.79, \mathrm{p}<0.0001\right)$. The following interaction effects were observed: TEC $\times H L D$ $\left(\mathrm{F}_{1,9}=295.41, \mathrm{p}<0.0001\right), T E C \times D\left(\mathrm{~F}_{2,18}=186.05\right.$, $\mathrm{p}<0.0001), H L D \times D\left(\mathrm{~F}_{2,18}=97.55, \mathrm{p}<0.0001\right), D \times$ $W\left(\mathrm{~F}_{4,36}=7.93, \mathrm{p}=0.0001\right)$, and TEC $\times H L D \times D$ $\left(\mathrm{F}_{2,18}=124.53, \mathrm{p}<0.0001\right)$. Repeated measure analysis of variance in $C A T 2$ showed a significant main effect for TEC $\left(\mathrm{F}_{1,9}=280.72, \mathrm{p}<0.0001\right), D\left(\mathrm{~F}_{3,27}=373.00\right.$, $\mathrm{p}<0.0001)$, and $W\left(\mathrm{~F}_{2,18}=284.19, \mathrm{p}<0.0001\right)$. The following interaction effects were observed: $T E C \times D$ $\left(\mathrm{F}_{3,27}=63.52, \mathrm{p}<0.0001\right)$, and $D \times W\left(\mathrm{~F}_{6,54}=8.53\right.$, p $<0.0001$ ). Fig. 9 shows interaction plots of $T E C \times H L D$ by $D$, where GL $=\mathrm{GLH}+\mathrm{GLV}, \mathrm{GP}=\mathrm{GPH}+\mathrm{GPV}$, $\mathrm{AL}=\mathrm{ALH}+\mathrm{ALV}$, and $\mathrm{AP}=\mathrm{APH}+\mathrm{APV}$ for $D=100,200$ and 400 , and $\mathrm{GL}=\mathrm{GLH}, \mathrm{GP}=\mathrm{GPV}, \mathrm{AL}=\mathrm{ALH}$, and $\mathrm{AP}=\mathrm{APV}$ for $D=600$. $N U M$ increases for $D=400$ and 600 in GL and GP, and on the other hand, $N U M$ stays around 1.0 even for longer $D$ in AL and AP. This means that the single dragging operation achieves to reach longer distance targets by the proposed technique. In addition, we observe different behaviors of GL and GP at $D=400$ and 600 in Fig. 9. This result implies that the movable range of the tip of an index finger would be slightly different; the range of the portrait orientation grip style would be larger than that of the landscape orientation. As a result, the mean $N U M$ of the generic technique increased, and on the other hand, that of the proposed technique was not deteriorated even for longer $D$. 


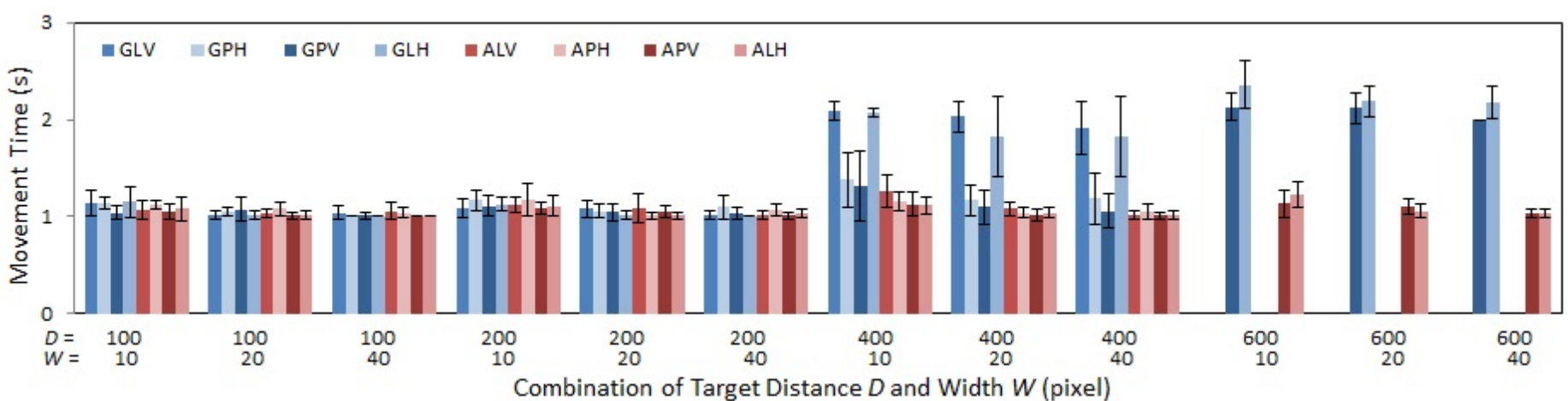

Fig. 8: The number of dragging by $W$ and $D$ for eight models

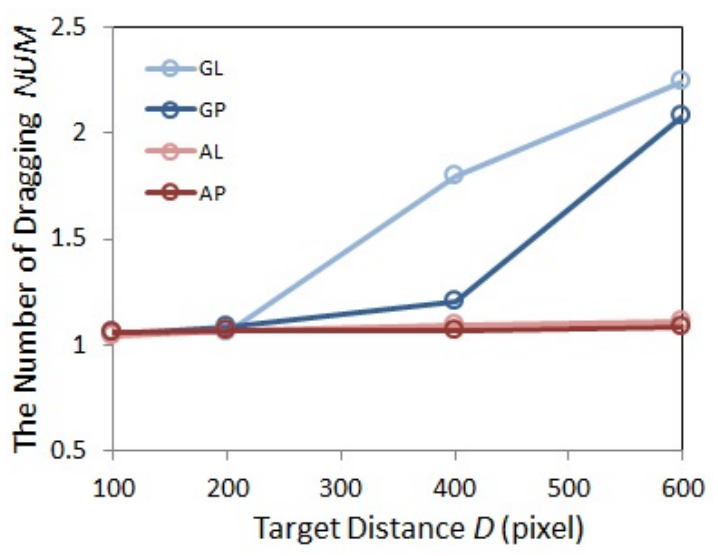

Fig. 9: $N U M$ by $D$ for four models $(T E C \times H L D)$

\section{DISCUSSION}

Results in Fig. 4 show that plots of GLV, GPH, ALV and $\mathrm{APH}$ are aligned on a straight line and yielded $r^{2}$ values of above 0.95 . Although step-wise plots caused by an increase of $N U M$ at $D=400$ and 600 are observed in GLH and GVP, we find better alignments of $r^{2}$ values of 0.945 in ALH and 0.978 in APV. The performance improvements of the proposed technique are clearly demonstrated in Fig. 7 and Fig. 9. Actually, the approach as addressed by the proposed technique is effective to utilize the limited range inputs. Results in Fig. 6 indicate that the longitudinal movement would be easier than the transverse movement of the tip of an index finger. While, results in Fig. 9 imply that the movable range of the tip of an index finger in the portrait orientation grip style would be slightly larger than that of the landscape orientation. These results suggest that APV would be the most appropriate design for single-handed user interactions using rear touch.

\section{CONClusion ANd Future Work}

We have conducted a basic study of single-handed cursor control technique optimized for rear touch operation, and showed the performance improvements in target selection tasks by the proposed technique in comparison with the generic technique. Especially, we demonstrated that the single dragging operation achieved to reach longer distance targets by the proposed technique. Since the movable area of the tip of an index finger on a rear touch panel is limited, the technique, such that small movement of dragging accomplishes long distance cursor movement, is effective. In addition, evaluation in relation to the way of holding the handheld device and the direction of dragging operation suggested the existence of advantageous combinations for usability. These results will be the beneficial knowledge for better design of single-handed user interactions using rear touch.

Considering a practical use of handheld devices, not only cursor navigation but also command execution, such as scroll or zoom, is required. To find the ways to control commands, which have an affinity for the combined use with rear-touchbased cursor navigation system, is an important future work. Touch-gesture-based approach [20], [21], [22] with regarding touch-pressure [23], [24] has potential for solutions. The influence of the size of display and the weight of device on performance is also of significant interest. And the effects of haptic feedback are another topic of future consideration for improvements of usability.

\section{REFERENCES}

[1] R. L. Potter, L. J. Weldon, and B. Shneiderman, "Improving the accuracy of touch screens: an experimental evaluation of three strategies," in Proceedings of the SIGCHI conference on Human factors in computing systems. ACM, 1988, pp. 27-32.

[2] A. K. Karlson and B. B. Bederson, "Thumbspace: generalized onehanded input for touchscreen-based mobile devices," in HumanComputer Interaction-INTERACT 2007. Springer, 2007, pp. 324-338.

[3] D. Vogel and P. Baudisch, "Shift: a technique for operating pen-based interfaces using touch," in Proceedings of the SIGCHI conference on Human factors in computing systems. ACM, 2007, pp. 657-666.

[4] A. Roudaut, S. Huot, and E. Lecolinet, "Taptap and magstick: improving one-handed target acquisition on small touch-screens," in Proceedings of the working conference on Advanced visual interfaces. ACM, 2008, pp. $146-153$.

[5] D. Wigdor, C. Forlines, P. Baudisch, J. Barnwell, and C. Shen, "Lucid touch: a see-through mobile device," in Proceedings of the 20th annual ACM symposium on User interface software and technology. ACM, 2007, pp. 269-278.

[6] P. Baudisch and G. Chu, "Back-of-device interaction allows creating very small touch devices," in Proceedings of the SIGCHI conference on Human factors in computing systems. ACM, 2009, pp. 1923-1932.

[7] T. Ohtani, T. Hashida, Y. Kakehi, and T. Naemura, "Comparison of front touch and back touch while using transparent double-sided touch display," in ACM SIGGRAPH 2011 Posters. ACM, 2011, p. 42. 
[8] M. Sugimoto and K. Hiroki, "Hybridtouch: an intuitive manipulation technique for pdas using their front and rear surfaces," in Proceedings of the 8th conference on Human-computer interaction with mobile devices and services. ACM, 2006, pp. 137-140.

[9] X.-D. Yang, E. Mak, P. Irani, and W. F. Bischof, "Dual-surface input: augmenting one-handed interaction with coordinated front and behindthe-screen input," in Proceedings of the 11th International Conference on Human-Computer Interaction with Mobile Devices and Services. ACM, 2009, p. 5.

[10] E.-1. E. Shen, S.-s. D. Tsai, H.-h. Chu, Y.-j. J. Hsu, and C.-w. E. Chen, "Double-side multi-touch input for mobile devices," in CHI'09 Extended Abstracts on Human Factors in Computing Systems. ACM, 2009, pp. 4339-4344.

[11] J. Scott, S. Izadi, L. S. Rezai, D. Ruszkowski, X. Bi, and R. Balakrishnan, "Reartype: text entry using keys on the back of a device," in Proceedings of the 12th international conference on Human computer interaction with mobile devices and services. ACM, 2010, pp. 171180.

[12] O. Schoenleben and A. Oulasvirta, "Sandwich keyboard: fast ten-finger typing on a mobile device with adaptive touch sensing on the back side," in Proceedings of the 15th international conference on Humancomputer interaction with mobile devices and services. ACM, 2013, pp. $175-178$.

[13] J. O. Wobbrock, B. A. Myers, and H. H. Aung, "The performance of hand postures in front-and back-of-device interaction for mobile computing," International Journal of Human-Computer Studies, vol. 66, no. 12 , pp. $857-875,2008$.

[14] M. Noor and M. Faizuddin, "Exploring back-of-device interaction," in Proceedings of the adjunct publication of the 26th annual ACM symposium on User interface software and technology. ACM, 2013, pp. $45-48$.

[15] S. S. A. Shimon, S. Morrison-Smith, N. John, G. Fahimi, and J. Ruiz, "Exploring user-defined back-of-device gestures for mobile devices," in Proceedings of the 17th International Conference on Human-Computer Interaction with Mobile Devices and Services. ACM, 2015, pp. 227232.
[16] J. Wagner, S. Huot, and W. Mackay, "Bitouch and bipad: designing bimanual interaction for hand-held tablets," in Proceedings of the SIGCHI Conference on Human Factors in Computing Systems. ACM, 2012, pp. 2317-2326.

[17] Y. Guiard, "Asymmetric division of labor in human skilled bimanual action: The kinematic chain as a model," Journal of motor behavior, vol. 19, no. 4, pp. 486-517, 1987.

[18] P. M. Fitts, "The information capacity of the human motor system in controlling the amplitude of movement." Journal of experimental psychology, vol. 47, no. 6, p. 381, 1954.

[19] I. S. MacKenzie, "Fitts' law as a research and design tool in humancomputer interaction," Human-computer interaction, vol. 7, no. 1, pp. 91-139, 1992.

[20] F. Wang and X. Ren, "Empirical evaluation for finger input properties in multi-touch interaction," in Proceedings of the SIGCHI Conference on Human Factors in Computing Systems. ACM, 2009, pp. 1063-1072.

[21] A. Bragdon, E. Nelson, Y. Li, and K. Hinckley, "Experimental analysis of touch-screen gesture designs in mobile environments," in Proceedings of the SIGCHI Conference on Human Factors in Computing Systems. ACM, 2011, pp. 403-412.

[22] K. Wolf, C. Müller-Tomfelde, K. Cheng, and I. Wechsung, "Pinchpad: performance of touch-based gestures while grasping devices," in Proceedings of the Sixth International Conference on Tangible, Embedded and Embodied Interaction. ACM, 2012, pp. 103-110.

[23] C. Harrison, J. Schwarz, and S. E. Hudson, "Tapsense: enhancing finger interaction on touch surfaces," in Proceedings of the 24th annual ACM symposium on User interface software and technology. ACM, 2011, pp. 627-636.

[24] S. Boring, D. Ledo, X. Chen, N. Marquardt, A. Tang, and S. Greenberg, "The fat thumb: using the thumb's contact size for single-handed mobile interaction," in Proceedings of the 14th international conference on Human-computer interaction with mobile devices and services. ACM, 2012, pp. 39-48. 